\title{
Development of a real-time TaqMan PCR assay for the detection of porcine circovirus 4
}

\author{
Wanting Chen ${ }^{1,2,3^{*}}$, Dike Jiang ${ }^{1,2,3^{*}}$, Lu Xiao $^{3 *}$, \\ Pengfei Zhang ${ }^{2,3}$, Yan $\mathrm{Luo}^{2,3}$, Zexiao Yang, ${ }^{2,3}$, Xueping $\mathrm{Yao}^{2,3}$, \\ Yin Wang ${ }^{2,3}$, Xulong $\mathrm{Wu}^{1,1}$ \\ ${ }^{1}$ Chengdu Agricultural College, Chengdu 611130, China \\ ${ }^{2}$ College of Veterinary Medicine, Sichuan Agricultural University, Chengdu 611130, China \\ ${ }^{3}$ Key Laboratory of Animal Diseases and Human Health of Sichuan Province, College of Veterinary Medicine, \\ Sichuan Agricultural University, Chengdu 611130, China \\ yaanwuxl@163.com
}

Received: July 1, 2021 Accepted: January 11, 2022

\begin{abstract}
Introduction: Porcine circovirus 4 (PCV4) was first discovered in 2019 in a herd of pigs with porcine respiratory disease, dermatitis and nephropathy syndrome in Hunan Province, China. It has subsequently been detected in other provinces and in South Korea. In consideration of the potential of the virus to cause an epidemic, rapid, sensitive, and specific detection of PCV4 is needed, as is the facilitation of further epidemiological research through elucidation of the whole genome of PCV4. This study had those two aims. Material and Methods: Fifty-five blood samples, two pig tissue samples, nine saliva swabs and one semen sample which all originated from Sichuan province pig farms were analysed. The virus' genome of 1,770 bp was synthesised artificially based on a Chinese reference strain and primers and probes for the ORF2 gene were designed. Then, the amplified target fragment was cloned into the pMD19-T vector and a series of diluted recombinant plasmids were used to generate a standard curve. An optimised real-time TaqMan PCR method was established. Results: The results of this study showed that the established method is specific for PCV4 but not for other viruses, and has amplification efficiency of $99.6 \%$, a regression squared value $\left(\mathrm{R}^{2}\right)$ of 1.000 and a detection limit of $2.2 \times 10$ DNA copies. This method was shown to be analytically specific and sensitive with a low intra- and inter-assay coefficient of variation $(<1.67 \%)$. Of a total of 67 clinical samples tested using the established method, three were shown to be positive (4\%). Conclusion: This study confirms the existence of PCV4 in Sichuan and provides a promising alternative tool for rapid detection of PCV4.
\end{abstract}

Keywords: porcine circovirus 4, real-time TaqMan PCR, detection method.

\section{Introduction}

Porcine circovirus (PCV) is a circular singlestranded negative-strand DNA non-enveloped virus that belongs to the Circoviridae family and the Circovirus genus (7). Currently, four different porcine circoviruses have been reported: PCV1 (16), PCV2 (8), PCV3 (10) and PCV4 (20). The first of the viruses was discovered in 1974 as a contaminant of a porcine kidney cell line (PK-15) and is considered non-pathogenic (15). On the other hand, PCV2 is recognised as a necessary but insufficient agent of porcine-circovirus-associated diseases (PCVAD), including postweaning multisystemic wasting syndrome (PMWS) and porcine dermatitis and nephropathy syndrome (PDNS) $(5,12,17)$. PCV2 has become a threat to the swine industry globally. In 2016 , a new porcine circovirus was detected from the tissue homogenate of sows exhibiting similar symptoms to PDNS and inflammation of the heart and multiple organs, and the virus, designated PCV3, was identified to be another potential pathogenic factor of clinical diseases in pigs $(10,11)$.

In April 2019 in Hunan Province, China, Zhang et al. (20) discovered a new type of PCV in pigs with severe clinical diseases, including respiratory diseases, diarrhoea, porcine dermatitis and nephrotic syndrome; this type, PCV4, poses a threat to the pig industry. The genome of PCV4 has a total length of 1,770 nucleotides (nt). Based on prediction using ORFfinder (https://www.ncbi.nlm.nih.gov/orffinder/), PCV4 contains 
12 open reading frames (ORF) with two major predicted genes: a replicase gene spanning $891 \mathrm{nt}$ encoding a protein of 296 amino acids, and a capsid gene spanning $687 \mathrm{nt}$ encoding a protein of 228 amino acids (20). There were no relevant reports of PCV4 detection in other regions, and therefore an efficient, sensitive and rapid detection method is needed to provide specific diagnosis that may facilitate accurate clinical investigation in minimal time during the PCV4 epidemic.

\section{Material and Methods}

Viruses and clinical samples. All viruses used in this study were collected in our laboratories, including porcine reproductive and respiratory syndrome virus (PRRSV) strains, porcine epidemic diarrhoea virus (PEDV), classical swine fever virus (CSFV) attenuated vaccine, porcine parvovirus (PPV) and pseudorabies virus (PRV). A total of 67 samples (55 blood samples, 2 tissue samples, 9 saliva swabs and 1 semen sample) were investigated, which were submitted to the Department of Veterinary Medicine, Sichuan Agricultural University from 15 pig farms located in the cities of Chengdu, Mianyang, Suining, Dazhou, Zigong, Dazhou, Nanchong, and Guangyuan of the Sichuan province of China between 2018 and 2021.

Construction of the PCV4 whole genome plasmid. The whole genome of PCV4 (1,770 bp) was synthesised artificially (Sangon Biotech, Shanghai, China) based on a reference sequence of a Chinese isolate available in GenBank (accession no. MT311852.1), and then cloned into pUC57 Vector (TIANGEN, Beijing, China). The plasmid DNA construct of pUC57-PCV4 was amplified in E. coli DH5 $\alpha$ and subsequently purified using a Plasmid MiniPrep Kit (Sangon Biotech) and quantified using a NanoDrop 2000 spectrophotometer (Thermo Fisher, Waltham, MA, USA).

Primers and probe for PCV4. Forward and reverse PCV4 primers and a PCV4 probe were designed manually based on the conserved regions shown by the alignment of the ORF2 gene from complete genome sequences of the PCV4 reference sequence isolated in China. The nucleotide sequences of the primers and the probe are listed in Table 1.

DNA cloning and sequencing. The plasmid constructed in the previous step was used as the template for DNA amplification using the PCV4-F/R primer set. The amplified target fragment was recovered and then cloned into pMD-19T Vector (TaKaRa, Dalian, China) for DH5 $\alpha$ (TIANGEN) transformation. Following colony screening and identification, positive clones containing the desired DNA insert were grown overnight in a culture volume of $5 \mathrm{~mL}$ for plasmid isolation using a Plasmid MiniPrep Kit (Sangon Biotech). The DNA concentration of the plasmid construct pMD19T-PCV4 was quantified using the NanoDrop 2000 spectrophotometer (Thermo Scientific). The number of plasmid DNA copies was calculated using the following formula: amount $($ copies $/ \mu \mathrm{L})=($ DNA concentration $(\mathrm{g} / \mu \mathrm{L}) /($ plasmid length in base pairs $\times 660) \times 6.02 \times 10^{23}$.

Optimisation of the real-time PCR. A real-time PCR was performed on the CFX Connect Real-Time PCR Detection System (Bio-Rad, Hercules, CA, USA) in a reaction volume of $25 \mu \mathrm{L}$ containing $12.5 \mu \mathrm{L}$ of SsoAdvanced Universal Probe Supermix (Bio-Rad), different volumes of each primer $(10 \mu \mathrm{mol} / \mathrm{L})$ and probe (10 $\mu \mathrm{mol} / \mathrm{L}), 2 \mu \mathrm{L}$ of DNA template and RNase-free deionised distilled water $\left(\mathrm{ddH}_{2} \mathrm{O}\right)$. The qPCR conditions were as follows: initial denaturation at $94^{\circ} \mathrm{C}$ for $2 \mathrm{~min}$ followed by 40 cycles at $94^{\circ} \mathrm{C}$ for $10 \mathrm{~s}$ and final extension at $55.4^{\circ} \mathrm{C}$ for $30 \mathrm{~s}$.

Method standardisation. The DNA concentration of the pMD19T-PCV4 plasmid construct was determined using the NanoDrop2000 spectrophotometer (Thermo Scientific) before being serially diluted ten-fold. Each diluted concentration in the range from $2.20 \times 10^{8}$ copies $/ \mu \mathrm{L}$ to $2.20 \times 10^{3}$ copies $/ \mu \mathrm{L}$ was tested by qPCR in triplicate. The calibration curves were generated by CFX Manager software (Bio-Rad). The standard curve of $\mathrm{Ct}$ versus plasmid copies was then constructed.

Specificity analysis. For specificity analysis, $10 \mathrm{ng}$ of viral DNA or cDNA from PCV2, PCV3, PPV, PRV, PRRSV, PEDV, FMDV vaccine and CSFV vaccine (all provided by the Sichuan Agricultural University) was used as templates for amplification by real-time PCR. The analysis also used pMD19T-PCV4 plasmid construct as the positive control and $\mathrm{ddH}_{2} \mathrm{O}$ as the negative control. To evaluate the specificity of this method, the real-time TaqMan PCR assay established in this study was used for amplification.

Sensitivity analysis. Plasmid DNA of pMD19PCV4 with a concentration ranging from $10^{8}$ to $10^{1}$ copies/ $\mu \mathrm{L}$ was used to evaluate the sensitivity of the real-time PCR. The best reaction conditions with the minimum detection volume in the PCR assay developed in the course of this investigation were used for sensitivity evaluation.

Table 1. Nucleotide sequences of PCV4 primers and PCV4 probe

\begin{tabular}{lll}
\hline Primer & Nucleotide sequence $\left(5^{\prime}-3^{\prime}\right)$ & Nt position \\
\hline PCV4-F & AATCTCACTGTCCACACCTG & $1105-1124$ \\
PCV4-R & CAAAACCCCAGGACCCATC & $1267-1249$ \\
PCV4-probe & FAM-ACCCACACCCTCCACTTCCAGC-BHQ1 & \\
\hline
\end{tabular}

PCV4 - porcine circovirus 4 
Repeatability and reproducibility analyses. To determine the reproducibility and repeatability of the real-time PCR, $10^{5}, 10^{4}, 10^{3}$ and $10^{2}$ copies of pMD19PCV4 were tested in triplicate in three independent experiments. The inter-assay variability was evaluated in three independent runs and in different thermocyclers (ABI, Forster, CA, USA; Roche Diagnostics, RischRotkreuz, Switzerland; Bio-Rad) performed on different days. The mean, standard deviation and coefficient of variation $(\mathrm{CV})$ were calculated using the $\mathrm{Ct}$ values obtained for each dilution in each run. The range (minimum and maximum values) for each parameter was determined. The mean, standard deviation and the CV were calculated using SPSS statistical software (IBM, Armonk, NY, USA) to evaluate the method repeatability.

Detection of PCV4 from clinical samples. Viral DNA was extracted from 72 clinical samples, which were amplified using the developed real-time PCR method. Samples amplified before 38 cycles with typical amplification curves were considered PCV4-positive. Evaluation was repeated if a typical amplification curve was observed with a $\mathrm{Ct}$ value of greater than 38 . Samples that underwent repeated tests with the same result were considered positive, and those showing inconsistent results in repeated tests were considered negative.

\section{Results}

Real-time PCR optimisation. Reactions containing $12.5 \mu \mathrm{L}$ of SsoAdvanced Universal Probe Supermix (Bio-Rad), $8.05 \mu \mathrm{L}$ of RNase-free $\mathrm{ddH}_{2} \mathrm{O}$, $2 \mu \mathrm{L}$ of DNA template, $1.1 \mu \mathrm{L}$ of each primer $(10 \mu \mathrm{mol} / \mathrm{L})$ and $0.25 \mu \mathrm{L}$ of solution of probe (10 $\mu \mathrm{mol} / \mathrm{L})$ were found to produce the strongest fluorescent signals.

Using the pUC57-PCV4 construct as the template, the target fragment with a length of $163 \mathrm{bp}$ was successfully amplified by PCR with specific primers. Following DNA purification, the PCR products were cloned into the pMD19-T Vector to construct a pMD19T-PCV4 recombinant plasmid standard. Comparison of the DNA sequencing results for the isolates from the samples with the reference sequence revealed no mutation in the sequence of the DNA insert, showing that the pMD19T-PCV4 recombinant plasmid standard had been successfully constructed. DNA quantification showed that the plasmid concentration was $2.20 \times 10^{9}$ copies $/ \mu \mathrm{L}$.

Standard curve. For standard curve generation, pMD19T-PCV4 was diluted tenfold in RNase-free $\mathrm{ddH}_{2} \mathrm{O}$, and the diluted samples were used as the template for the real-time PCR. As shown in Fig. 1, the generated standard curve had a range of $10^{3}-10^{8}$ copies $/ \mu \mathrm{L}$ with a good linear correlation between $2.20 \times 10^{8}$ copies $/ \mu \mathrm{L}$ and $2.20 \times 10^{3}$ copies $/ \mu \mathrm{L}$ (Slope: -3.330 ; y-int: 34.697; correlation coefficient $\mathrm{R}^{2}$ : 1.000; amplification efficiency: $99.6 \%$ ).

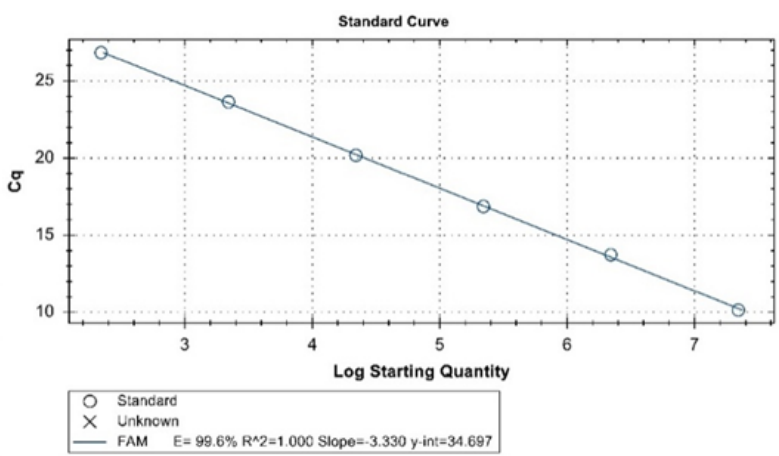

Fig. 1. Standard curve of PCV4 real-time PCR for serially diluted pMD19T-PCV4 recombinant plasmids. Mean threshold cycle $(\mathrm{Ct})$ values from three replicates (y-axis) are plotted versus logarithmic concentrations of plasmid copies ( $\mathrm{x}$-axis)

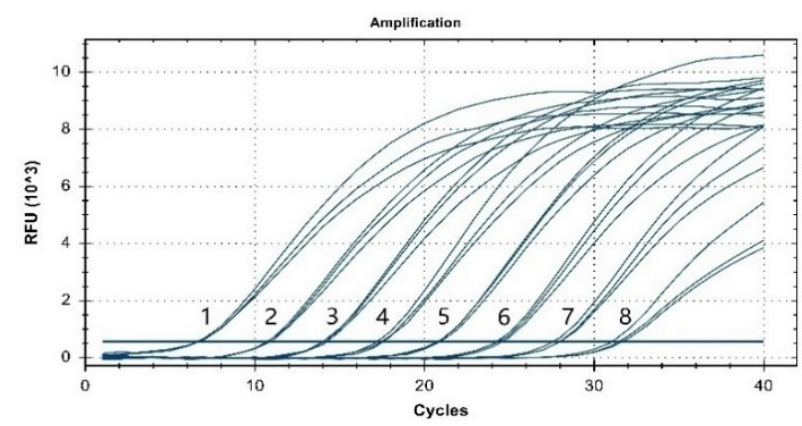

Fig. 2. Sensitivity analysis of the TaqMan real-time PCR for PCV4. RFU - relative fluorescence units; $1-8-2.2 \times 10^{8}$ copies $/ \mu \mathrm{L}-2.2 \times 10^{1}$ copies $/ \mu \mathrm{L}$. The lowest copy number detected by qPCR was $2.2 \times 10^{1}$ copies $/ \mu \mathrm{L}$

Specificity analysis. Using DNA and cDNA of other viruses or vaccines as templates, our results showed that only PCV4 was detected by real-time PCR, while other viruses were undetected. Similar results were obtained from three repeated independent reactions, demonstrating that the assay was highly specific.

Sensitivity analysis. To evaluate the assay sensitivity, diluted pMD19T-PCV4 samples with a concentration range of $10^{8}-10^{1}$ copies $/ \mu \mathrm{L}$ were used as a template. Our results showed that the real-time PCR had a detection limit of $10^{1}$ copies and similar results were obtained from three repeated independent reactions (Fig. 2).

Reproducibility analysis. In order to evaluate the repeatability of the detection method, the assay was carried out using recombinant plasmids at three different concentrations from $2.20 \times 10^{5}$ to $2.20 \times 10^{2} \mathrm{copies} / \mu \mathrm{L}$. Each concentration was evaluated three times, and the determined $\mathrm{Ct}$ values, mean, standard deviation and $\mathrm{CV}$ are shown in Table 2. Our results showed that the experimentally established method for the detection of PCV4 genotype by fluorescence quantitative PCR has good reproducibility. Therefore, this method can be used for stable and reliable detection of the PCV4 genotype from the samples.

Detection of PCV4 in clinical samples. Using 67 samples collected from pig farms in different regions of the Sichuan province from 2018 to 2021, the practicability of the established quantitative PCR 
(qPCR) detection method based on TaqMan was evaluated. A positive rate of $4 \%(3 / 67)$ was determined by qPCR, all samples positive by qPCR were sequenced. The similarity between MT311852.1 and the four isolates ranged from $95.45 \%$ to $98.85 \%$.

Table 2. Repeatability test of qPCR

\begin{tabular}{lcccc}
\hline $\begin{array}{l}\text { Positive } \\
\text { plasmid } \\
\text { concentration }\end{array}$ & \multicolumn{2}{c}{ Intra-assay } & & Inter-assay \\
& & & & \\
\hline$($ copies $/ \mu \mathrm{L})$ & $($ mean $\pm \mathrm{SD})$ & $\begin{array}{c}\mathrm{CV} \\
\%\end{array}$ & $\begin{array}{c}\mathrm{Ct} \\
(\text { mean } \pm \mathrm{SD})\end{array}$ & $\begin{array}{c}\mathrm{CV} \\
\%\end{array}$ \\
$2.20 \times 10^{5}$ & $18.15 \pm 0.22$ & 1.21 & $18.14 \pm 0.25$ & 1.38 \\
$2.20 \times 10^{4}$ & $21.48 \pm 0.14$ & 0.65 & $21.49 \pm 0.18$ & 0.84 \\
$2.20 \times 10^{3}$ & $24.98 \pm 0.16$ & 0.64 & $24.92 \pm 0.28$ & 1.12 \\
$2.20 \times 10^{2}$ & $28.56 \pm 0.36$ & 1.26 & $28.53 \pm 0.48$ & 1.68 \\
\hline
\end{tabular}

$\mathrm{Ct}$ - threshold cycle

\section{Discussion}

The four PCVs are similar in structure with two ORFs oriented in opposite directions in the circular genome; ORF1 or the rep genes Rep and Rep' encoding proteins associated with replication (not characterised for PCV3 or PCV4 at this point); and ORF2 or the cap gene encoding the capsid or Cap protein (1). The fourth type of the virus was first identified in 7- and 12-weekold pigs suffering from respiratory, enteric and PDNS symptoms, and also in healthy pigs in April 2019 (20). Since the discovery of PCV4, only a few sequences of the virus have become available and the clinical significance and pathogenic mechanism of the virus have not been reported. An efficient and stable detection method is an effective means to control and prevent the spread of the newly emerging virus.

Currently, real-time fluorescent PCR is widely used in clinical detection. Compared with loop-mediated isothermal amplification, the TaqMan-based real-time PCR assay has superior accuracy. Isothermal amplification requires specialised personnel for operation, and the samples are vulnerable to contamination; therefore, the potential for false positive results is high and it is difficult to guarantee the accuracy of detection (18). Compared with SYBR, the cost of TaqMan assays is higher, where the expense probe synthesis particularly is a consideration. Another of their disadvantages is that quantification is often affected by enzyme performance because of the use of the $5^{\prime} \rightarrow 3^{\prime}$ exonuclease activity of the Taq enzyme. However, the advantage of real-time TaqMan PCR over SYBRGreen-based PCR (19) is that it yields highly specific, low false positives and read-through signals generated by the TaqMan probe. Compared with conventional PCR (14), real-time PCR is able to detect the reaction process in real time and determine the absolute amount of amplified products, with strong specificity and better sensitivity. The developed real-time PCR assay is rapid with no requirement for post-PCR steps. Real-time TaqMan PCR and ELISA are the most commonly used laboratory confirmation methods for the early diagnosis of the virus, but the targets detected by the two methods are different. Viral antibody detection is an indirect method of confirming viral infections, so the clinical significance is slightly different. In viral infections, viraemia occurs first, and then the antibodies are produced. The real-time TaqMan PCR method can detect positive specimens $\leq 1 \mathrm{~d}$ after the onset, while the ELISA method can detect positive samples only a few days after (4).

At present, according to research reports, PCV4 has been detected in Hunan, Guangxi, Shaanxi, Henan and Inner Mongolia provinces of China (3, 6, 13, 14, 20). This is the first study to report the successful detection of PCV4 in the Sichuan province. The virus was also successfully detected in South Korea in 2021 (9). Evidence is lacking of this virus' presence outside Asia: PCV4 has not been detected in Italy or Spain (2). It may be due to trade in pork products between these two countries and China not taking place frequently. However, PCV4 may eventually circulate globally because of international trade.

In conclusion, we successfully developed and evaluated a real-time PCR assay for rapid and accurate detection of PCV4. Our established real-time PCR method detected down to a lower limit of 101 copies, and it was analytically specific and sensitive with low intra- and inter-assay CV ( $<1.67 \%)$, indicating reproducible and reliable in providing rapid and accurate PCV4 detection.

* Wanting Chen and Dike Jiang contributed equally to this work.

Conflict of Interests Statement: The authors declare that there is no conflict of interests regarding the publication of this article.

Financial Disclosure Statement: This work was supported by the following grants: Scientific Researching Fund Projects of Chengdu Agricultural College in 2020 (20ZR101) and the Chengdu Technological Innovation Research and Development Project in 2020 (2019-YF05-00119-SN).

Animal Rights Statement: The tissue samples in this study were collected from clinically diseased pigs with the approval of the local centre of animal disease control, and animal experiments were not conducted, therefore an animal rights statement is not applicable.

Acknowledgements: The authors would like to thank reviewers for their comments and suggestions which greatly improved the manuscript from its original version.

\section{References}

1. Finsterbusch T., Steinfeldt T., Caliskan R., Mankertz A.: Analysis of the subcellular localization of the proteins Rep, Rep' and Cap 
of porcine circovirus type 1 . Virology $2005,343,36-46$, doi: 10.1016/j.virol.2005.08.021.

2. Franzo G., Ruiz A., Grassi L., Sibila M., Drigo M., Segalés J.: Lack of Porcine circovirus 4 Genome Detection in Pig Samples from Italy and Spain. Pathogens 2020, 9, 433, doi: 10.3390/pathogens9060433.

3. Ha Z., Yu C., Xie C., Wang G., Zhang Y., Hao P., Li J., Li Z., Li Y., Rong F., Nan F., Zhang H., Zhuang X., Xie Y., Shi N., Lu H., Jin N.: Retrospective surveillance of porcine circovirus 4 in pigs in Inner Mongolia, China, from 2016 to 2018. Arch Virol 2021, 166, 1951-1959, doi: 10.1007/s00705-021-05088-w.

4. Haley C., Wagner B., Puvanendiran S., Abrahante J., Murtaugh M.P.: Diagnostic performance measures of ELISA and quantitative PCR tests for porcine circovirus type 2 exposure using Bayesian latent class analysis. Prev Vet Med 2011, 101, 79-88, doi: 10.1016/j.prevetmed.2011.05.001.

5. Harding J.C.: The clinical expression and emergence of porcine circovirus 2. Vet Microbiol 2004, 98, 131-135, doi: 10.1016/j.vetmic.2003.10.013.

6. Hou C.-Y., Zhang L.-H., Zhang Y.-H., Cui J.-T., Zhao L., Zheng L.-L., Chen H.-Y.: Phylogenetic analysis of porcine circovirus 4 in Henan Province of China: A retrospective study from 2011 to 2021. Transbound Emerg Dis 2021, doi: 10.1111/tbed.14172.

7. Mankertz A., Caliskan R., Hattermann K., Hillenbrand B., Kurzendoerfer P., Mueller B., Schmitt C., Steinfeldt T., Finsterbusch T.: Molecular biology of Porcine circovirus: analyses of gene expression and viral replication. Vet Microbiol 2004, 98, 81-88, doi: 10.1016/j.vetmic.2003.10.014.

8. Nayar G.P., Hamel A., Lin L.: Detection and characterization of porcine circovirus associated with postweaning multisystemic wasting syndrome in pigs. Can Vet J 1997, 38, 385-386.

9. Nguyen V.-G., Do H.-Q., Huynh T.M., Park Y.H., Park B.K., Chung H.C.: Molecular-based detection, genetic characterization and phylogenetic analysis of porcine circovirus 4 from Korean domestic swine farms. Transbound Emerg Dis 2021, doi: 10.1111/tbed.14017.

10. Palinski R., Piñeyro P., Shang P., Yuan F., Guo R., Fang Y., Byers E., Hause B.M.: A Novel Porcine Circovirus Distantly Related to Known Circoviruses Is Associated with Porcine Dermatitis and Nephropathy Syndrome and Reproductive Failure. J Virol 2016, 91, e01879-16, doi: 10.1128/JVI.01879-16.

11. Phan T.G., Giannitti F., Rossow S., Marthaler D., Knutson T.P., Li L., Deng X., Resende T., Vannucci F., Delwart E.: Detection of a novel circovirus PCV3 in pigs with cardiac and multi- systemic inflammation. Virol J 2016, 13, 184, doi: 10.1186/s12985-016-0642-z.

12. Segalés J., Calsamiglia M., Olvera A., Sibila M., Badiella L., Domingo M.: Quantification of porcine circovirus type 2 (PCV2) DNA in serum and tonsillar, nasal, tracheo-bronchial, urinary and faecal swabs of pigs with and without postweaning multisystemic wasting syndrome (PMWS). Vet Microbiol 2005, 111, 223-229, doi: 10.1016/j.vetmic.2005.10.008.

13. Sun W., Du Q., Han Z., Bi J., Lan T., Wang W., Zheng M.: Detection and genetic characterization of porcine circovirus 4 (PCV4) in Guangxi, China. Gene 2021, 773, 145384, doi: 10.1016/j.gene.2020.145384.

14. Tian R.-B., Zhao Y., Cui J.-T., Zheng H.-H., Xu T., Hou C.-Y., Wang Z.-Y., Li X.-S., Zheng L.-L., Chen H.-Y.: Molecular detection and phylogenetic analysis of Porcine circovirus 4 in Henan and Shanxi Provinces of China. Transbound Emerg Dis 2021, 68, 276-282, doi: 10.1111/tbed.13714.

15. Tischer I., Gelderblom H., Vettermann W., Koch M.A.: A very small porcine virus with circular single-stranded DNA. Nature 1982, 295, 64-66, doi: 10.1038/295064a0.

16. Tischer I., Rasch R., Tochtermann G.: Characterization of papovavirus-and picornavirus-like particles in permanent pig kidney cell lines. Zentralbl Bakteriol Orig A 1974, 226, 153-167.

17. Turner M.J., Medley G.F., Woodbine K.A., Slevin J.A., Green L.E.: The relationship between porcine circovirus 2 antigen score and antibody titre and histology of lymph nodes in 375 euthanased sick and healthy pigs from 113 British pig farms with and without postweaning multisystemic wasting syndrome. Prev Vet Med 2009, 88, 213-219, doi: 10.1016/j.prevetmed.2008.11.001.

18. Wang Y., Li Y., Cui Y., Jiang S., Liu G., Wang J., Li Y.: Establishment of a duplex SYBR green I-based real-time polymerase chain reaction assay for the rapid detection of canine circovirus and canine astrovirus. Molecular Cell Probes 2020, 54, 101666, doi: 10.1016/j.mcp.2020.101666.

19. Zhang D., Bai C., Ge K., Li Y., Gao W., Jiang S., Wang Y.: Establishment of an SYBR Green-based real-time PCR assay for porcine circovirus type 4 detection. J Virol Methods 2020, 285, 113963, doi: 10.1016/j.jviromet.2020.113963.

20. Zhang H.-H., Hu W.-Q., Li J.-Y., Liu T.-N., Zhou J.-Y., Opriessnig T., Xiao C.-T.: Novel circovirus species identified in farmed pigs designated as Porcine circovirus 4, Hunan province, China. Transbound Emerg Dis 2020, 67, 1057-1061, doi: 10.1111/tbed.13446 